\title{
The role of cell-free DNA in fibrinolysis for intraventricular hemorrhage
}

\author{
*Fangke Xie, MD, ${ }^{1}$ Qiang Tan, MD, ${ }^{2}$ Anyong Yu, MD, PhD, ${ }^{1}$ Peiwen Guo, MD, ${ }^{2}$ Ling Wang, MD, ${ }^{2}$ \\ Zongwei Zeng, MD, ${ }^{2}$ Liang Liang, MD, ${ }^{2}$ Jishu Xian, MD, ${ }^{2}$ Hua Feng, MD, PhD, ${ }^{2}$ and \\ Zhi Chen, MD, PhD²
}

'Department of Emergency, Affiliated Hospital of Zunyi Medical University, Zunyi; and 'Department of Neurosurgery, Southwest Hospital, Third Military Medical University (Army Medical University), Chongqing, China

\begin{abstract}
OBJECTIVE Tissue plasminogen activator (tPA) fibrinolysis did not improve functional outcomes of patients with intraventricular hemorrhage (IVH), largely because of the unsatisfactory clot clearance. The presence of neutrophil extracellular traps (NETs) within the clot has been confirmed to impair tPA fibrinolysis, but the mechanism has been unclear. The authors hypothesized that cell-free DNA (cfDNA), the main framework of NETs, might be the important reason for the fibrinolysis resistance, and they validated the hypothesis, hoping to provide a new target to promote intraventricular fibrinolysis.

METHODS First, cfDNA was detected in IVH clots by immunofluorescence staining in a rat model of IVH. Second, after blood (with or without exogenous cfDNA) intraventricular injection, IVH rats were given intraventricular infusion of $2 \mu$ l of saline, tPA, or tPA + DNase1 randomly. Then, the ventricular volume, animal behavior, and reactive astrocyte proliferation were assessed. Third, the IVH clots were collected for fibrinolysis assay in vitro. Finally, the effects of exogenous cfDNA in IVH were evaluated.
\end{abstract}

RESULTS The presence of cfDNA in clots was observed as early as 1 hour after IVH. Compared with the whole-blood model, blood + cfDNA caused more severe ventricular dilation (day 7: blood $32.47 \pm 2.096 \mathrm{~mm}^{3}$ vs blood + DNA $40.09 \pm$ $2.787 \mathrm{~mm}^{3}, p<0.05$ ), increased fibrinolysis resistance to tPA (day 7: tPA + DNA $26.04 \pm 1.318 \mathrm{~mm}^{3}$ vs tPA $22.15 \pm 1.706$ $\mathrm{mm}^{3}, \mathrm{p}<0.05$ ), and further deteriorated the functional defects in rats (blood vs blood + DNA, $\left.p<0.05\right)$. Degradation of cfDNA by DNase1 further enhanced the fibrinolysis effects on relieving the ventricular dilation (day 7: tPA + DNase1 $11.67 \pm 2.023 \mathrm{~mm}^{3}$ vs tPA, $p<0.05$ ), improving the functional outcome (tPA vs tPA + DNase1, $p<0.05$ ) and reducing periventricular astrocyte proliferation.

CONCLUSIONS cfDNA impaired tPA fibrinolysis for IVH, and degradation of cfDNA may be a new target to improve this condition.

https://thejns.org/doi/abs/10.3171/2020.7.JNS201429

KEYWORDS intraventricular hemorrhage; tissue plasminogen activator; neutrophil extracellular traps; cell-free DNA; fibrinolysis; vascular disorders

\begin{abstract}
A $\mathrm{s}$ a global disease with high morbidity and mortality, intraventricular hemorrhage (IVH) has always been the research focus in the field of neuroscience, but the current treatment remains very limited. ${ }^{1}$ External ventricular drainage (EVD) is a conventional method for IVH treatment, but simple drainage does not promote intraventricular clot resolution; improperly placed catheters or catheter blockage may even cause fatal intracranial infections. ${ }^{2,3}$ In recent years, intraventricular tissue plasminogen activator (tPA) fibrinolysis therapy for IVH has been considered a safe and promising treatment. ${ }^{4,5}$ However,
\end{abstract}

fibrinolysis therapy did not improve functional outcomes of patients with IVH, largely because of unsatisfactory clot clearance. ${ }^{6,7}$ Many methods, including standardizing the surgical approaches and increasing the dosage of tPA, have been tried to promote the fibrinolytic effect, but none of them have worked. ${ }^{8-11}$ Therefore, investigation of a new target to improve fibrinolysis efficacy is needed.

Neutrophil extracellular traps (NETs) were first reported as the extracellular fibers released by activated neutrophils to kill bacteria. ${ }^{12}$ With more extensive research, NETs have been found to participate not only in the immune

ABBREVIATIONS cfDNA = cell-free DNA; DAPI = 4',6-diamidino-2-phenylindole; EVD = external ventricular drainage; GFAP = glial fibrillary acidic protein; H3Cit = citrullinated histone 3; ICP = intracranial pressure; IVH = intraventricular hemorrhage; mNSS = modified Neurological Severity Score; NET = neutrophil extracellular trap; tPA = tissue plasminogen activator.

SUBMITTED April 24, 2020. ACCEPTED July 21, 2020.

INCLUDE WHEN CITING Published online January 8, 2021; DOI: 10.3171/2020.7.JNS201429.

${ }^{*}$ F.X., Q.T., and A.Y. contributed equally to this work. 
defense, but also in the noninfectious process, including, but not limited to, thrombosis and fibrinolysis. ${ }^{13,14}$ NETs were observed in ischemic and hemorrhagic stroke clots, contributing to the fibrinolytic resistance, ${ }^{15,16}$ but the exact mechanism was not clear. Cell-free DNA (cfDNA) could exert antifibrinolytic effects via 1) enhancing the stability of the clots by providing a mesh scaffold for platelet and red blood cell adhesion, ${ }^{13}$ 2) competing for plasmin with fibrin, ${ }^{17}$ and 3) accelerating the inactivation of tPA by plasminogen activator inhibitor- $1 .{ }^{17}$ As the main framework of NETs, cfDNA might be the key reason for the fibrinolysis resistance of clots to tPA. Therefore, we tested this hypothesis using a rat IVH model, hoping to provide a new target to promote intraventricular fibrinolysis.

\section{Methods}

\section{Animals}

Animal use protocols were approved by the Army Military Medical University Committee on the Use and Care of Animals. Excluding 4 rats that died during the surgery, 113 adult male Sprague Dawley rats (weight 250-300 g) from the Army Medical University were used in this study. Animals were housed in a controlled pathogen-free environment at a temperature of approximately $23^{\circ} \mathrm{C}-25^{\circ} \mathrm{C}, 70 \%$ humidity, with 12-hour light/dark cycles and free access to food and water. Animal experimental procedures were performed under the Regulations for the Administration of Affairs Concerning Experimental Animals approved by the State Council of the People's Republic of China.

\section{Experimental Design}

This study consisted of 4 parts. In part 1, 25 rats were injected intraventricularly with arterial blood to induce IVH. At $0.5,1,1.5,6$, and 12 hours after blood injection, the brains (5 per each time point) were collected for cfDNA detection in the IVH clots by immunofluorescence staining.

In part 2, to explore the effects of cfDNA on tPA fibrinolysis for IVH, 40 rats were randomly divided into the following 5 groups (8 rats per group): sham, vehicle, tPA, tPA + DNase1, and tPA + DNA. The sham group only required a needle injection into the right ventricle. The vehicle group, tPA group, and tPA + DNasel group were given saline, tPA, and tPA + DNase1, respectively, 1 hour after intraventricular injection of whole blood. ${ }^{18,19}$ In the tPA + DNA group, the blood + DNA mixtures were injected into the right ventricle, followed by tPA fibrinolysis 1 hour later. On days 1,3 , and 7 , the degree of ventricular dilation was measured by MRI, and changes in animal behavior were analyzed. In addition, the reactive proliferation of astrocytes around the ipsilateral periventricular tissue was also assessed on day 7.

In part 3, to further explore the effect of cfDNA on tPA fibrinolysis in vitro, 24 rats were injected intraventricularly with blood or blood + DNA mixtures to induce IVH, and the clots were collected and weighed for fibrinolysis assay 3 days later.

For part 4, the effect of exogenous cfDNA in IVH was evaluated. Twenty-four rats were randomly assigned to 3 groups (8 rats per group). Group 1 received DNA solu- tions, group 2 received blood, and group 3 received blood + DNA mixtures; then the degree of ventricular dilation and animal behavior were assessed.

\section{IVH Model}

IVH was induced according to the previously described method. ${ }^{20}$ Rats were anesthetized with pentobarbital (40 $\mathrm{mg} / \mathrm{kg}$, intraperitoneally). Body temperature was maintained at $37^{\circ} \mathrm{C}$ by a feedback-controlled heating pad. Rats were positioned in a stereotactic frame (Kopf Instruments), and a 1-mm burr hole was drilled on the skull $1.6 \mathrm{~mm}$ lateral to the midline. A 29-gauge needle was inserted stereotactically into the right ventricle (coordinates: $0.6 \mathrm{~mm}$ posterior, $4.5 \mathrm{~mm}$ ventral, $1.6 \mathrm{~mm}$ lateral to the bregma). The 200- $\mu$ l aliquots of calf thymus DNA solution ( $2 \mu \mathrm{g}$; Macklin Biochemical), autologous whole blood, or blood + DNA mixtures ( $2 \mu \mathrm{g}$ of DNA was mixed with the whole blood) were injected at a rate of $20 \mu \mathrm{l} / \mathrm{min}$ using a microinfusion pump. The dose of the exogenous DNA was selected according to related fibrinolysis experiments and was within the safe dose range. ${ }^{21,22}$ After injection, the needle was removed, the burr hole was filled with bone wax, and the skin incision was closed with sutures. The animals were placed in a $37^{\circ} \mathrm{C}$ incubator to recover. The sham-operated animals only required a needle injection into the right ventricle.

\section{cfDNA Detection}

Because costaining of citrullinated histone 3 (H3Cit) and 4',6-diamidino-2-phenylindole (DAPI) has been used as a biomarker of NETs, ${ }^{23,24}$ the DNA colabeled with H3Cit can be regarded as extracellular DNA, i.e., cfDNA. Rats were anesthetized with pentobarbital $(100 \mathrm{mg} / \mathrm{kg}$, intraperitoneally) and perfused with $4 \%$ paraformaldehyde at $0.5,1,1.5,6$, and 12 hours after intraventricular injection of autogenous arterial blood. Then, the brains were collected, fixed, embedded, dehydrated, and sliced successively. For immunofluorescence staining, the slices were incubated with the primary antibodies of mouse anti-histone H3 (citrulline R2 + R8 + R17; 1:100, Abcam) overnight at $4^{\circ} \mathrm{C}$, and were probed subsequently with appropriate secondary antibodies for 2 hours at $37^{\circ} \mathrm{C}$. The DNA was counterstained with DAPI (Boster Biological Technology). For SYTOX Orange staining, the slices were stained with $5 \mu \mathrm{M}$ SYTOX Orange (Molecular Probes, Inc. $)^{25}$ at room temperature for 10 minutes. Immunofluorescence was examined and captured using a confocal fluorescence microscope (LSM780, Zeiss). ${ }^{15}$

\section{Intraventricular Fibrinolysis}

According to published studies, ${ }^{16,26}$ equal amounts of saline $(2 \mu \mathrm{l})$, tPA $(10 \mu \mathrm{g} / \mu \mathrm{l}$; Boehringer Ingelheim International), and tPA + DNase1 (tPA $10 \mu \mathrm{g} / \mu \mathrm{l}$, DNase1 1000 $\mathrm{IU} / \mu \mathrm{l})$ were injected into the ventricle (coordinates: 0.6 $\mathrm{mm}$ posterior, $4.5 \mathrm{~mm}$ ventral, $1.6 \mathrm{~mm}$ lateral to the bregma) through a microinjection pump 1 hour after IVH. The sham-operated animals only required a needle injection into the right ventricle.

\section{MRI and Volume Measurement}

On days 1,3, and 7 after IVH, rats underwent MRI ex- 
amination with a 7.0-T Varian MR scanner (Bruker BioSpin) as described previously. ${ }^{19,27}$ Rats were anesthetized with a $2 \%$ isoflurane/air mixture throughout MRI examination. A T2 fast spin-echo sequence in a field of view of $35 \times 35 \mathrm{~mm}$ and 20 coronal slices (1.0-mm thickness) was used. The volumes of ipsilateral and contralateral ventricles were calculated by combining the total areas and multiplying by the thickness $(1 \mathrm{~mm})$ using an image analysis system (ImageJ, NIH). Volume measurements were performed in a blinded manner.

\section{Behavioral Test}

For behavioral tests, all animals were tested before MRI and scored by experimenters who were blinded to the groups. Two tests were used. The first test was the modified Neurological Severity Score (mNSS). The mNSS method was used to assess the neurological function of experimental rats as previously described. ${ }^{19,28}$ This test (normal score $=0$, maximal deficit score $=18$ ), including motor tests, observation of abnormal movements, sensory tests, and reflex tests, was performed before IVH and on days 1,3 , and 7 after surgery.

The second test used was the forelimb placement score. ${ }^{19,28}$ The number of times that the rat placed the appropriate forelimb on the tabletop in response to whisker stimulation was recorded. Each rat was tested 10 times per forelimb, and the percentage of the appropriate forelimb placements was calculated.

\section{Glial Fibrillary Acidic Protein Staining}

Seven days after IVH, the rats were anesthetized and perfused and the brain was removed. The brain tissue was routinely fixed, dehydrated, embedded, sectioned, and blocked. The slices were incubated with the primary antibodies of mouse anti-glial fibrillary acidic protein (GFAP; 1:100, Proteintech) overnight at $4^{\circ} \mathrm{C}$ and were probed subsequently with appropriate secondary antibodies for 2 hours at $37^{\circ} \mathrm{C}$. Finally, the slices were counterstained with DAPI. Immunofluorescence was examined and captured using a confocal fluorescence microscope (LSM780, Zeiss). ${ }^{29}$ Six rats in each group were used for GFAP immunofluorescence staining. According to our previous method, ${ }^{30}$ two sections at approximately $3.3 \mathrm{~mm}$ behind the bregma of each animal were used to evaluate the proliferation of astrocytes near the ventricular wall. The GFAP-immunofluorescence-positive area was outlined and measured using ImageJ. Corresponding statistical analysis and histograms were generated using GraphPad Prism version 7.0 software.

\section{In Vitro IVH Clot Fibrinolysis Assay}

In the autologous blood-induced IVH groups, the IVH rats were anesthetized and killed by cervical dislocation on day 3. According to the method of Mirzadeh et al., ${ }^{31}$ the brain was removed and the subventricular zone tissue was separated to fully expose the lateral ventricle. We removed the clots within the ventricles and weighed them immediately. The fresh clots were randomly incubated in $1 \mathrm{ml}$ of saline, tPA $(1 \mu \mathrm{g} / \mathrm{ml})$, and tPA + DNasel (tPA 1 $\mu \mathrm{g} / \mathrm{ml}$, DNase1 $100 \mathrm{IU} / \mathrm{ml})$. Six rats were given intraven- tricular injection of the blood + DNA mixtures, and then the clots were collected and incubated in $1 \mathrm{ml}$ of tPA (1 $\mu \mathrm{g} / \mathrm{ml}$ ) on day 3. Clots were weighed at baseline ( $0 \mathrm{~min})$ and after 10,30,60, 90, and 120 minutes, and the degree of fibrinolysis was expressed as a percentage relative to baseline weight. ${ }^{16,32}$

\section{Statistical Analysis}

Statistical analysis was performed using GraphPad Prism version 7.0 software in a double-blind manner. Data were expressed as means \pm standard deviations. Comparisons of the weight of fresh clots between two groups were analyzed using 2-tailed Student t-tests. Other data for MRI, functional outcomes, GFAP staining, and in vitro IVH clot fibrinolysis assay were analyzed using 1-way ANOVA ( 1 factor) followed by the least significant difference test or the Newman-Keuls test. A p value $<0.05$ was considered statistically significant.

\section{Results cfDNA in IVH Clots}

The confocal images of costaining of H3Cit and DAPI showed that cfDNA began to appear as early as 1 hour after IVH and exhibited an increasing trend until 12 hours (Fig. 1A). Therefore, we chose the specimens of 12 hours postoperation for SYTOX Orange staining and found that long stringlike cfDNA structures were visible (Fig. 1B), further proving the presence of cfDNA.

\section{cfDNA Impaired tPA Fibrinolysis for IVH in Rats}

The volumes of lateral ventricles were assessed by T2weighted MRI on days 1, 3, and 7 after IVH (Fig. 2A). In the blood-induced IVH groups, tPA significantly reduced the lateral ventricular dilation (day 1: tPA 23.06 \pm $1.684 \mathrm{~mm}^{3}$ vs vehicle $37.28 \pm 2.441 \mathrm{~mm}^{3}, \mathrm{p}<0.05$; day 3: tPA $22.35 \pm 2.103 \mathrm{~mm}^{3}$ vs vehicle $35.29 \pm 1.688 \mathrm{~mm}^{3}$, $\mathrm{p}<0.05$; day 7: tPA $22.15 \pm 1.706 \mathrm{~mm}^{3}$ vs vehicle 33.31 $\pm 2.222 \mathrm{~mm}^{3}, \mathrm{p}<0.05$; Fig. $2 \mathrm{~B}$ ), and this effect was enhanced by degradation of cfDNA using DNasel (day 1: tPA + DNasel $12.40 \pm 1.884 \mathrm{~mm}^{3}$ vs tPA, $\mathrm{p}<0.05$; day 3: tPA + DNase1 $12.11 \pm 1.677 \mathrm{~mm}^{3}$ vs tPA, $\mathrm{p}<0.05$; day 7: tPA + DNase1 $11.67 \pm 2.023 \mathrm{~mm}^{3}$ vs tPA, $\mathrm{p}<0.05$; Fig. 2B). Compared with the blood-induced IVH model, the fibrinolytic effect of tPA on relieving ventricular dilation was partly counteracted in the blood + DNA IVH model (day 1: tPA + DNA $29.73 \pm 1.956 \mathrm{~mm}^{3}$ vs tPA, p $<0.05$; day 3: tPA + DNA $28.52 \pm 1.370 \mathrm{~mm}^{3}$ vs tPA, $\mathrm{p}<0.05$; day 7: tPA + DNA $26.04 \pm 1.318 \mathrm{~mm}^{3}$ vs tPA, $\mathrm{p}<0.05$; Fig. 2B), further suggesting that cfDNA impaired tPA fibrinolysis for IVH.

The increased mNSS scores and decreased forelimb placing scores in the vehicle group indicated that IVH led to neurological deficits (Fig. 3). In mNSS scores, tPA improved the functional outcome from day 1 to day 7 after IVH (tPA vs vehicle, $p<0.05$; Fig. 3A), which was further enhanced by addition of DNasel (tPA vs tPA + DNasel, $p<0.05$; Fig. 3A). Adding exogenous cfDNA weakened the effect of tPA on functional improvement (tPA vs tPA + DNA, p < 0.05; Fig. 3A). In the forelimb placing test on day 7 , the tPA group also showed significant functional 
Xie et al.
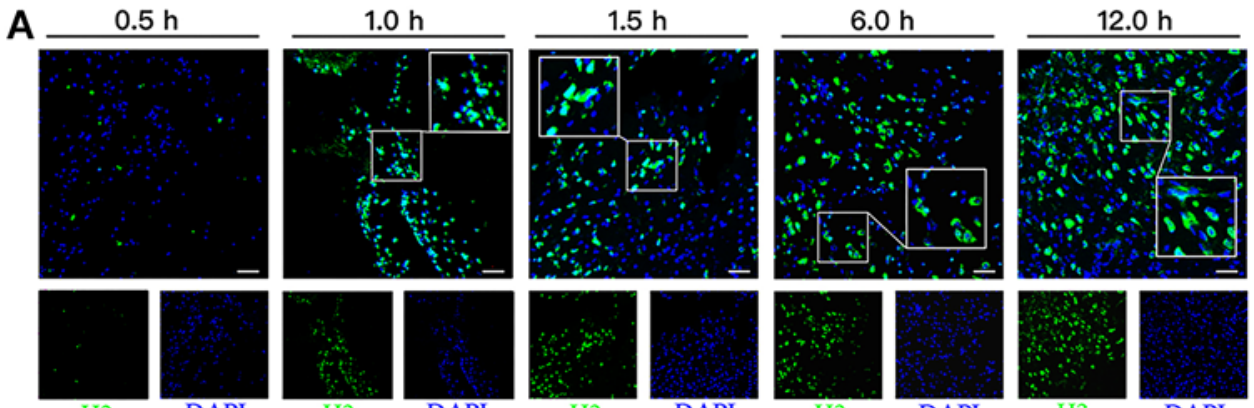

$\mathrm{H3}$
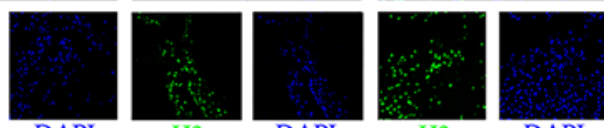

$\mathrm{H} 3$

DAPI

DAPI
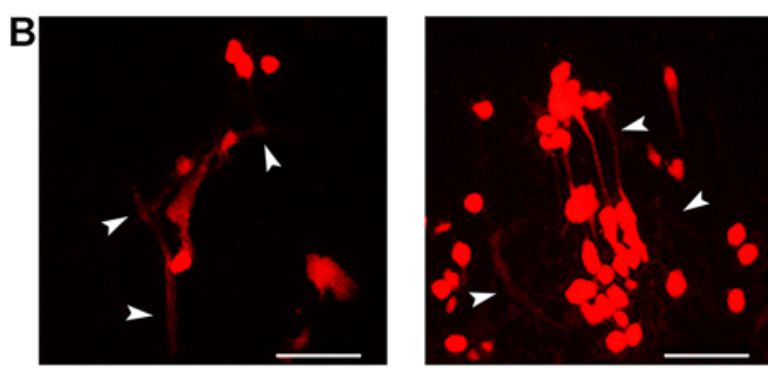

FIG. 1. cfDNA was present in IVH clots. A: Representative photographs of immunofluorescence staining for cfDNA (DAPI [blue] colabeled with $\mathrm{H} 3$ Cit [green]) in the clots at $0.5,1.0,1.5,6$, and 12 hours after IVH ( $n=5 /$ group). Each white square represents the respective magnified inset (below). Bar $=50 \mu \mathrm{m}$. B: Representative long stringlike cfDNA structures (arrowheads) by SYTOX Orange nucleic acid stain (orange). Bar $=50 \mu \mathrm{m}$. Figure is available in color online only.
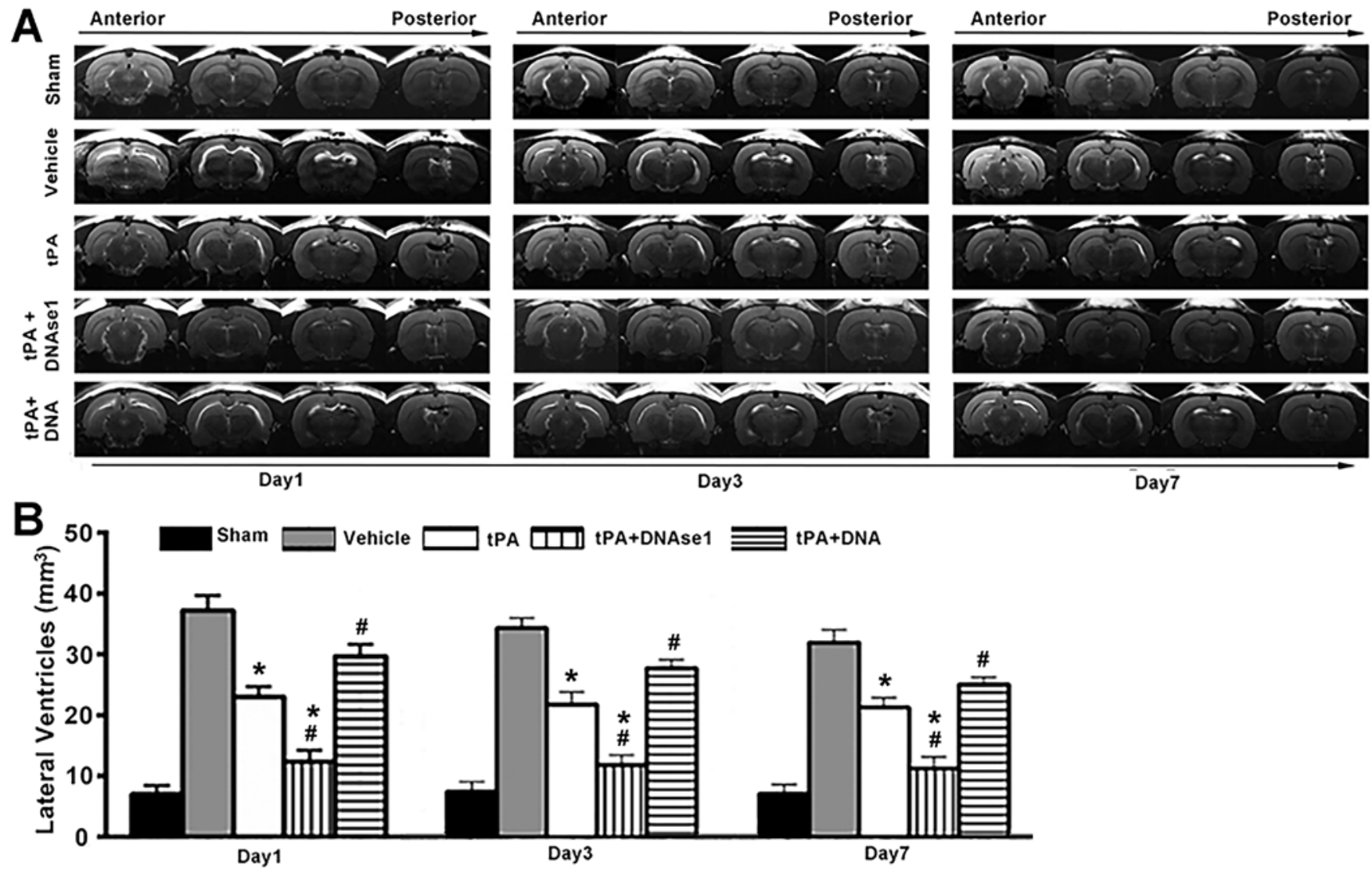

FIG. 2. Degradation of cfDNA by DNase1-enhanced tPA induced the reduction of ventricular dilation. A: Representative T2weighted MR images obtained on days 1, 3, and 7 after IVH ( $n=5 /$ group). B: Quantification of ventricular volumes according to the related T2-weighted images on days 1,3 , and 7 . Results are presented as means \pm SDs. ${ }^{*} p<0.05$ versus the vehicle group, ${ }^{\#}<<$ 0.05 versus the tPA group. 


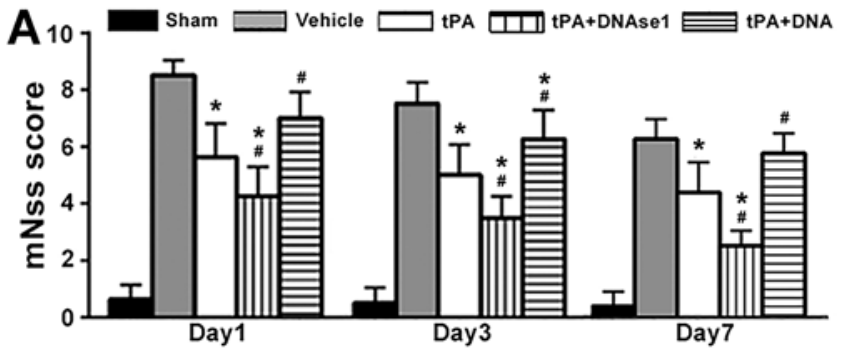

B

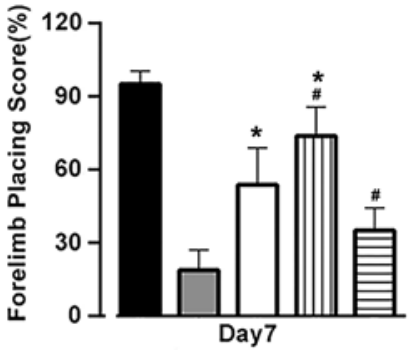

FIG. 3. Degradation of cfDNA by DNase1-enhanced tPA induced neurological function improvement. A: mNSS on days 1,3 , and 7 post-IVH ( $n=8$ /group). B: Forelimb placing test score on day 7 post-IVH $(n=8 /$ group). Results are presented as means \pm SDs. ${ }^{*} p<0.05$ versus the vehicle group, ${ }^{*} p<0.05$ versus the tPA group.

improvement (tPA vs vehicle, $p<0.05$; Fig. 3B), and additional DNase1 or cfDNA resulted in a strengthening or weakening trend on tPA effectiveness, respectively (tPA vs tPA + DNase1, $\mathrm{p}<0.05$; tPA vs tPA + DNA, $\mathrm{p}<0.05$; Fig. 3B).

The reactive proliferation of astrocytes was examined using immunofluorescence staining of GFAP. On day 7 after IVH, there was obvious reactive proliferation of as- trocytes in the ipsilateral periventricular tissue, which was reduced significantly by tPA fibrinolysis (tPA vs vehicle, $p<0.05$; Fig. 4). The combination of DNase1 could also reduce astrocyte proliferation (tPA + DNase1 vs vehicle, $p$ $<0.01$; Fig. 4), while additional cfDNA reversed the effect on alleviating the proliferation of astrocytes (tPA + DNA vs tPA, $\mathrm{p}<0.05$; Fig. 4).

\section{cfDNA Impairment of tPA-Induced Fibrinolysis In Vitro}

Three days after $200 \mu \mathrm{l}$ of autologous blood or blood + DNA intraventricular injection, the IVH clots were collected for tPA fibrinolysis assay in vitro. Compared with the blood groups, the clots from the blood + DNA group seemed larger (Fig. 5A) and weighed more (blood vs blood + DNA, p < 0.05; Fig. 5B). In the blood groups, tPA treatment effectively promoted fibrinolysis (residual weight to the initial weight at 120 minutes: blood + tPA vs blood + vehicle, $\mathrm{p}<0.05$; Fig. 5C). DNase1 significantly enhanced the tPA-induced fibrinolysis (120 minutes: blood + tPA + DNase1 vs blood + tPA, $p<0.05$; Fig. 5C). In contrast, the addition of cfDNA in IVH modeling resulted in a significant resistance to tPA fibrinolysis (blood + tPA vs blood + DNA + tPA, p $<0.05$; Fig. 5C).

\section{Effects of Exogenous cfDNA in IVH}

Compared with the sham group, injection of $200 \mu \mathrm{l}$ of DNA solution did not cause changes in ventricular volumes (sham vs DNA, $p>0.05$; Fig. 6) or animal behaviors (sham vs DNA, $p>0.05$; Fig. 7). However, intraventricular injection of blood + DNA mixtures resulted in more severe ventricular dilation (day 1: blood $36.18 \pm 2.169 \mathrm{~mm}^{3}$ vs blood + DNA $41.12 \pm 3.311 \mathrm{~mm}^{3}, \mathrm{p}<0.05$; day 3: blood $36.12 \pm 1.770 \mathrm{~mm}^{3}$ vs blood + DNA $39.84 \pm 2.159 \mathrm{~mm}^{3}, \mathrm{p}$
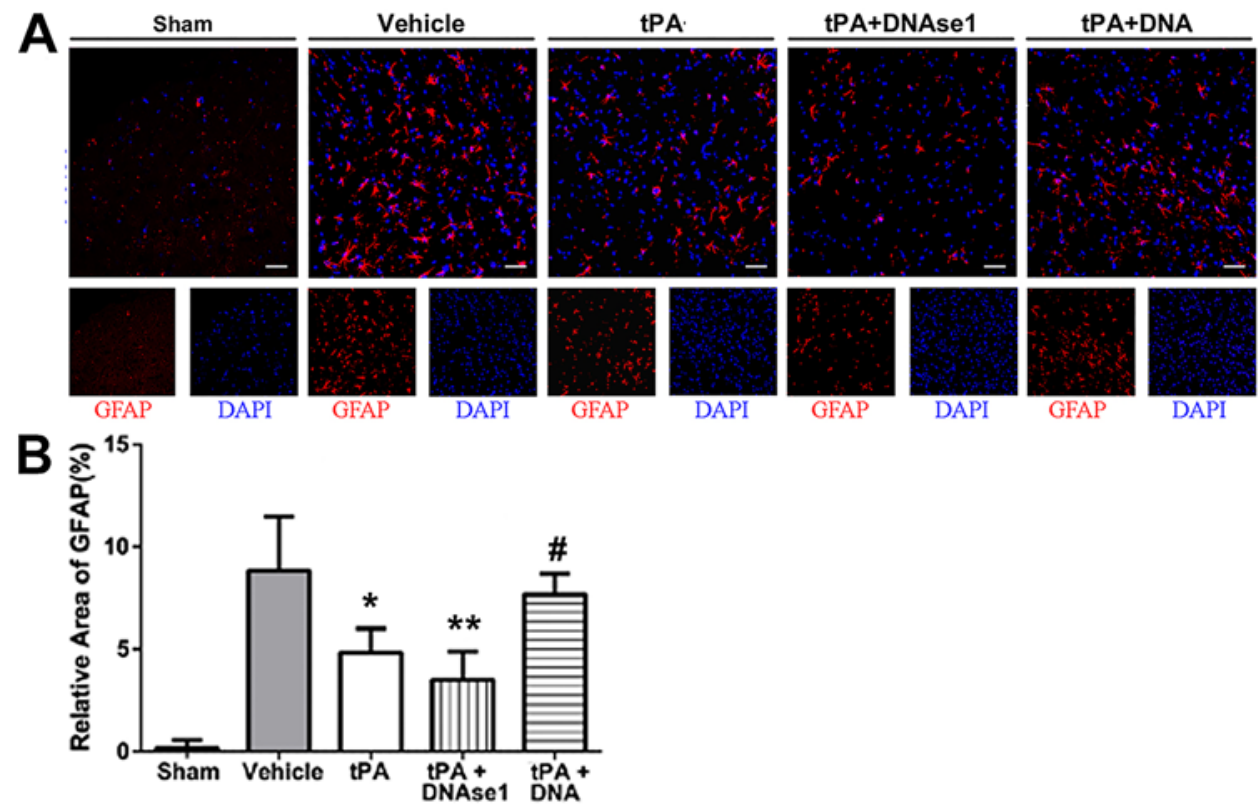

GFAP

DAPI

FIG. 4. Reactive proliferation of astrocytes around the ipsilateral periventricular tissue on day 7 after IVH. A: The reactive proliferation of astrocytes was examined using immunofluorescence staining of GFAP (red) and DAPI (blue). Bar = $50 \mu \mathrm{m}$. B: Quantification of the relative expression of GFAP ( $n=6 /$ group). Results are presented as means \pm SDs. ${ }^{\star} p<0.05$ versus the vehicle group, ${ }^{* *} p<0.01$ versus the vehicle group, ${ }^{*} p<0.05$ versus the tPA group. Figure is available in color online only. 
Xie et al.

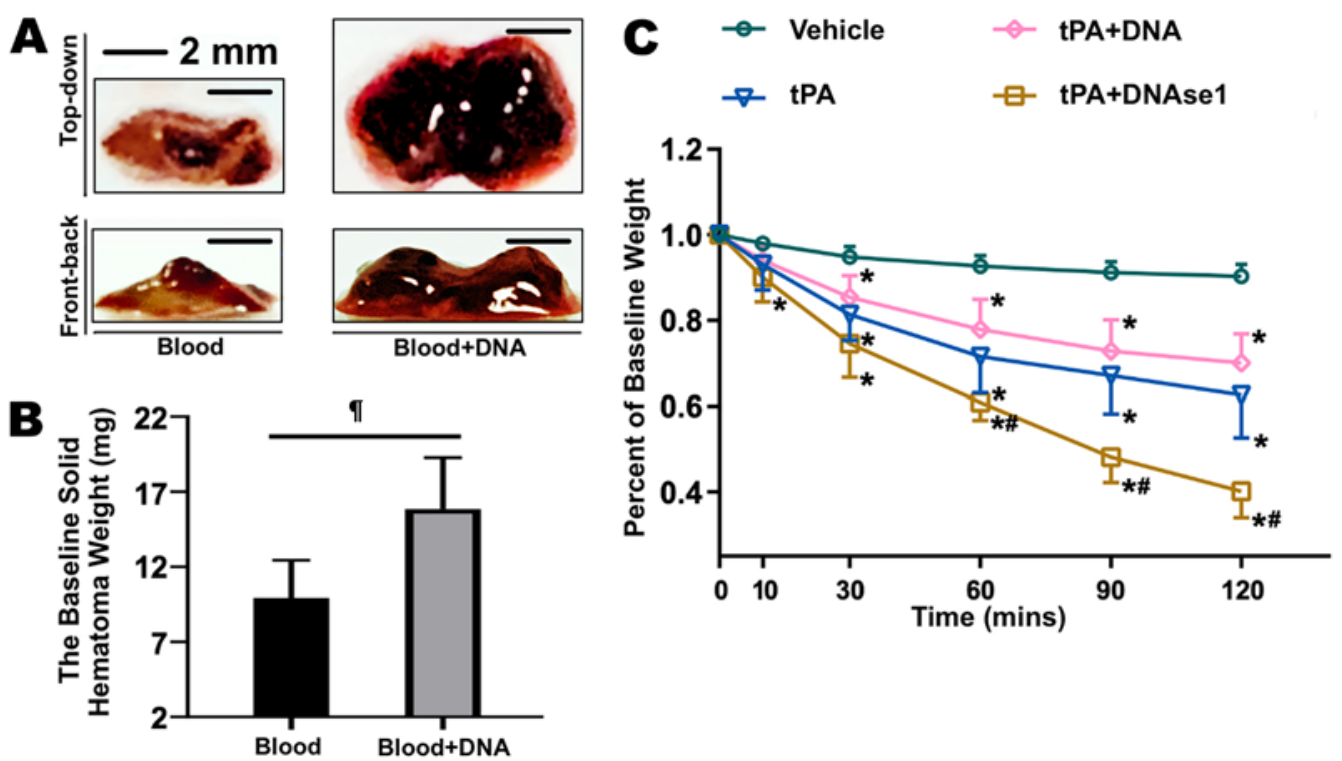

FIG. 5. cfDNA impaired tPA-induced clot fibrinolysis in vitro. A: Representations of the blood or blood + DNA clots collected from the ventricles of rats with IVH on day 3 . Bar $=2 \mathrm{~mm}$. B: Bar graph of the baseline solid clot weights ( $n=6 / g r o u p)$. Tp $<0.05 \mathrm{blood}$ versus blood + DNA. C: Results of the blood clots as a percentage baseline weight at different times ( $n=6 / g r o u p)$. Results are presented as means \pm SDs. ${ }^{*} p<0.05$ versus blood + vehicle, ${ }^{\#} p<0.05$ versus blood + tPA. Figure is available in color online only.
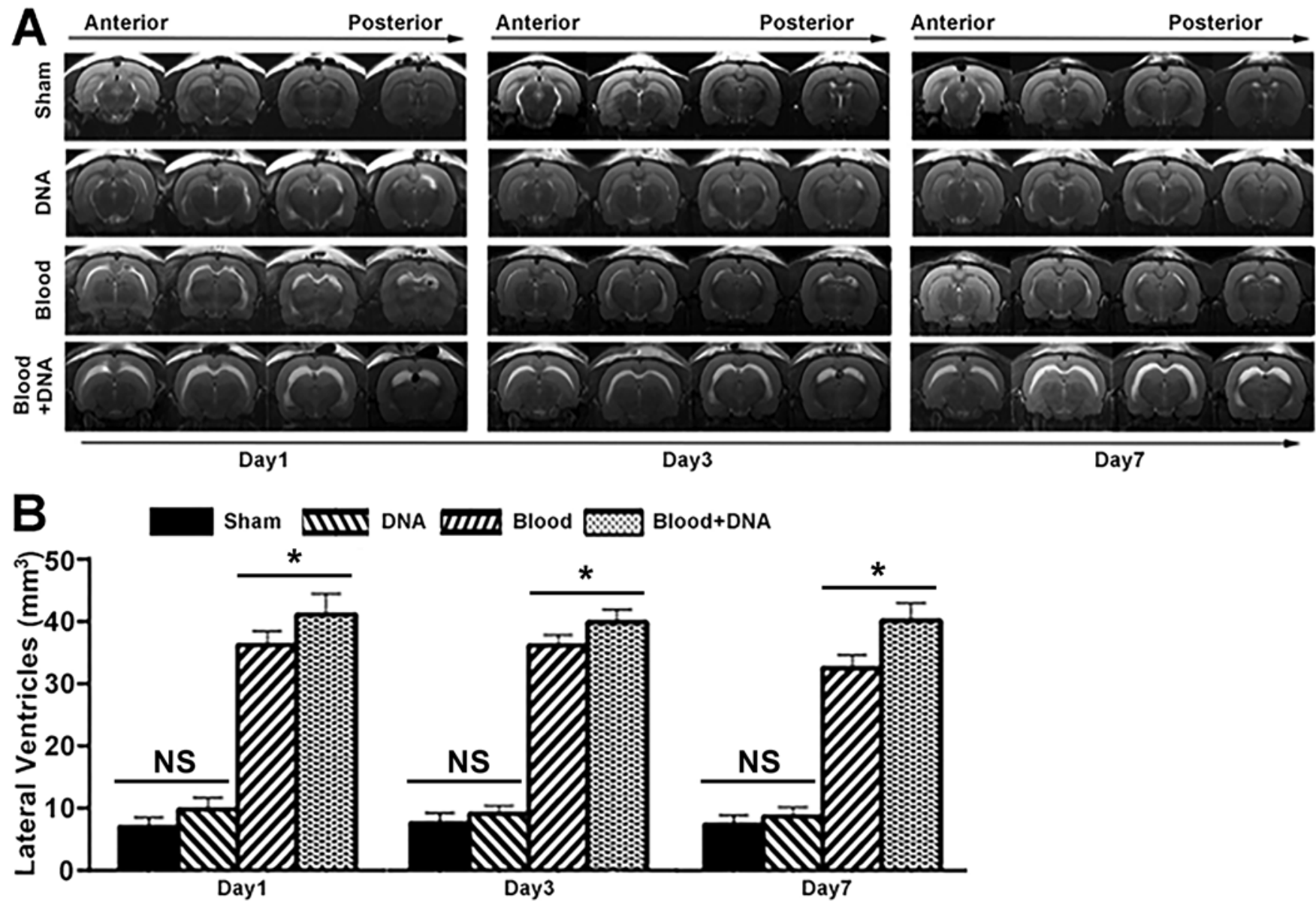

FIG. 6. The effect of exogenous cfDNA in ventricles. A: Representative T2-weighted images obtained on days 1, 3, and 7 after injection of blood or exogenous cfDNA ( $n=5 /$ group). B: Quantification of ventricular volumes according to the related T2-weighted MR images on days 1,3 , and 7 . Results are presented as means \pm SDs. ${ }^{*} p<0.05$, NS $=$ no significance. 


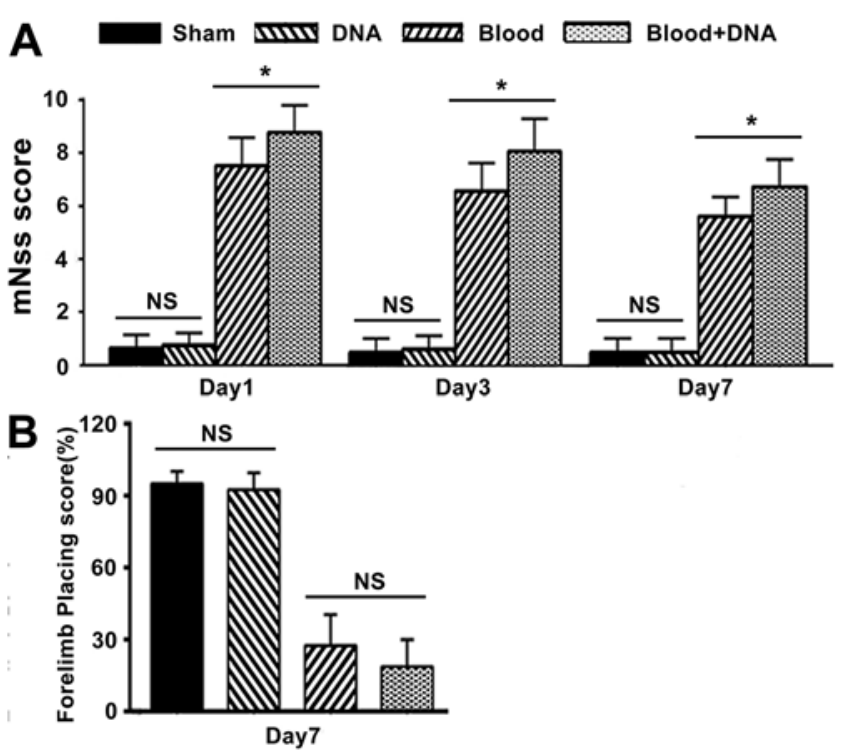

FIG. 7. The effect of exogenous cfDNA on behavior in rats. A: mNSS on days 1,3 , and 7 after injection of blood or exogenous cfDNA ( $n=8 /$ group). B: Forelimb placing test on day 7 after injection of blood or exogenous cfDNA ( $n=8 /$ group). Results are presented as means \pm SDs. ${ }^{*} p$ $<0.05, \mathrm{NS}=$ no significance.

$<0.05$; day 7: blood $32.47 \pm 2.096 \mathrm{~mm}^{3}$ vs blood + DNA $40.09 \pm 2.787 \mathrm{~mm}^{3}$, p < 0.05; Fig. 6) and worse functional outcome (blood vs blood + DNA, p $<0.05$; Fig. 7A) than that of blood injection alone.

\section{Discussion}

To our knowledge, this is the first study to explore the role of cfDNA in tPA fibrinolysis for IVH. We found that 1) cfDNA was present in IVH clots and impaired the fibrinolysis effect of tPA, and 2) degradation of cfDNA using DNase1 could improve tPA fibrinolysis, leading to a better outcome in rats with IVH.

By blocking the ventricular outflow and inducing a secondary inflammatory reaction, IVH often caused increased intracranial pressure (ICP), leading to devastating outcomes. ${ }^{33}$ Once obstructive hydrocephalus developed, EVD was commonly used to monitor ICP and assist with drainage of $\mathrm{CSF}^{2}$ However, simple drainage could not enhance the intrinsic thrombolytic activity of CSF and therefore could not promote intraventricular clot resolution. ${ }^{3}$ To accelerate intraventricular clot clearance, tPA fibrinolysis therapy emerged. Although tPA fibrinolysis therapy for IVH was safe and had lower case fatality at 6 months, it did not improve functional outcomes of IVH patients, ${ }^{7}$ and only a small number of patients achieved satisfactory clot clearance, which might be the main reason for the treatment failure. ${ }^{6}$ The previous clinical trials found that a higher dose of tPA administration could not promote fibrinolysis, but could increase the risk of rebleeding, leading to a worse functional outcome. ${ }^{7,34,35}$ In addition, the surgical approaches or different surgeons' experiences appeared irrelevant to the fibrinolysis efficacy. ${ }^{11,36}$ Because these methods did not improve tPA fibrinolysis, investigation of the structure and composition of clots may provide a new target.
NETs within the ischemic and hemorrhagic stroke clots have been found to impair tPA fibrinolysis, ${ }^{15,16}$ but the exact mechanism is not clear. The cfDNA that formed but was not limited in NETs showed great potential in antifibrinolytic effects. Because the surface of cfDNA has a high negative charge density, combined with its own double-stranded structure, cfDNA packed the fibrin network densely and hindered plasmin-mediated degradation of fibrin clots, leading to fibrinolysis impairment. ${ }^{13,37,38}$ In addition, cfDNA could compete for plasmin with fibrin and accelerate the inactivation of tPA by plasminogen activator inhibitor- $1 .{ }^{17}$ The presence of cfDNA in the IVH clot was observed in this study. Intraventricular injection of blood + cfDNA caused more severe ventricular dilation than that of blood injection alone. Although it is not obvious in terms of functional impairment, it may be related to our small sample size. This phenomenon might be explained by the hypothesis that the tangle of cfDNA made the clots more compact so that the endogenous or exogenous tPA could not play the full role of fibrinolysis, thus causing more lasting secondary injury. In addition, degradation of cfDNA by DNase1 enhanced the tPA fibrinolysis effects, further suggesting that cfDNA impaired tPA fibrinolysis for IVH.

\section{Limitations}

This study has some limitations. First, we did not set up a separate DNasel group during the drug administration group. Although in our previous studies ${ }^{6}$ we found that DNasel alone had no significant effect on the clearance of the hematoma, the addition of a DNase1 group will make the research more rigorous. Second, we only chose a 1-hour therapeutic time window to investigate the role of cfDNA in IVH fibrinolysis. Multiple time points should be added in future studies. Third, we used a surrogate of IVH in imaging ventricular dilation rather than ventricular blood to reflect the severity of IVH. The accurate and direct method to measure the ventricular volume and reflect the severity of IVH is worthy of further study.

\section{Conclusions}

In this study, we found that cfDNA was present in IVH clots and impaired the fibrinolysis effect. Degrading cfDNA using DNasel improved tPA fibrinolysis, leading to a better outcome in rats with IVH. This study provides a new target for fibrinolytic therapy for IVH that might have practical implications.

\section{Acknowledgments}

The present study was supported by the National Natural Science Foundation of China (grant no. 81771241 to Z.C.) and the Science and Technology Project of Guizhou Province (grant no. 5661, 2019).

\section{References}

1. Garton T, Hua Y, Xiang J, et al. Challenges for intraventricular hemorrhage research and emerging therapeutic targets. Expert Opin Ther Targets. 2017;21(12):1111-1122.

2. Dey M, Jaffe J, Stadnik A, Awad IA. External ventricular drainage for intraventricular hemorrhage. Curr Neurol Neurosci Rep. 2012;12(1):24-33. 
3. Carhuapoma JR. Thrombolytic therapy after intraventricular hemorrhage: do we know enough? J Neurol Sci. 2002;202(12):1-3.

4. Pang D, Sclabassi RJ, Horton JA. Lysis of intraventricular blood clot with urokinase in a canine model: Part 3. Effects of intraventricular urokinase on clot lysis and posthemorrhagic hydrocephalus. Neurosurgery. 1986;19(4):553-572.

5. Pang D, Sclabassi RJ, Horton JA. Lysis of intraventricular blood clot with urokinase in a canine model: Part 2. In vivo safety study of intraventricular urokinase. Neurosurgery. 1986;19(4):547-552.

6. Fitzgerald S. Alteplase for intraventricular hemorrhage is found to save lives, but not improve function. Neurol Today. 2017;17(7):10-11.

7. Hanley DF, Lane K, McBee N, et al. Thrombolytic removal of intraventricular haemorrhage in treatment of severe stroke: results of the randomised, multicentre, multiregion, placebocontrolled CLEAR III trial. Lancet. 2017;389(10069):603-611.

8. Wang YF, Tsirka SE, Strickland S, et al. Tissue plasminogen activator (tPA) increases neuronal damage after focal cerebral ischemia in wild-type and tPA-deficient mice. Nat Med. 1998;4(2):228-231.

9. Goto H, Fujisawa H, Oka F, et al. Neurotoxic effects of exogenous recombinant tissue-type plasminogen activator on the normal rat brain. $J$ Neurotrauma. 2007;24(4):745-752.

10. Wang YC, Lin CW, Shen CC, et al. Tissue plasminogen activator for the treatment of intraventricular hematoma: the dose-effect relationship. J Neurol Sci. 2002;202(1-2):35-41.

11. Fam MD, Hanley D, Stadnik A, et al. Surgical performance in minimally invasive surgery plus recombinant tissue plasminogen activator for intracerebral hemorrhage evacuation phase III clinical trial. Neurosurgery. 2017;81(5):860-866.

12. Brinkmann V, Reichard U, Goosmann C, et al. Neutrophil extracellular traps kill bacteria. Science. 2004;303(5663): 1532-1535.

13. Engelmann B. Extracellular DNA and histones as thrombus stabiliser. Thromb Haemost. 2015;113(6):1164.

14. Jorch SK, Kubes P. An emerging role for neutrophil extracellular traps in noninfectious disease. Nat Med. 2017;23(3): 279-287.

15. Ducroux C, Di Meglio L, Loyau S, et al. Thrombus neutrophil extracellular traps content impair tPA-induced thrombolysis in acute ischemic stroke. Stroke. 2018;49(3):754-757.

16. Tan Q, Guo P, Zhou J, et al. Targeting neutrophil extracellular traps enhanced tPA fibrinolysis for experimental intracerebral hemorrhage. Transl Res. 2019;211:139-146.

17. Gould TJ, Lysov Z, Liaw PC. Extracellular DNA and histones: double-edged swords in immunothrombosis. J Thromb Haemost. 2015;13(suppl 1):S82-S91.

18. Orakcioglu B, Becker K, Sakowitz OW, et al. MRI of the perihemorrhagic zone in a rat ICH model: effect of hematoma evacuation. Neurocrit Care. 2008;8(3):448-455.

19. Tan Q, Chen Q, Niu Y, et al. Urokinase, a promising candidate for fibrinolytic therapy for intracerebral hemorrhage. $J$ Neurosurg. 2017;126(2):548-557.

20. Chen Q, Tang J, Tan L, et al. Intracerebral hematoma contributes to hydrocephalus after intraventricular hemorrhage via aggravating iron accumulation. Stroke. 2015;46(10): 2902-2908.

21. Longstaff C, Varjú I, Sótonyi P, et al. Mechanical stability and fibrinolytic resistance of clots containing fibrin, DNA, and histones. J Biol Chem. 2013;288(10):6946-6956.

22. Varjú I, Longstaff C, Szabó L, et al. DNA, histones and neutrophil extracellular traps exert anti-fibrinolytic effects in a plasma environment. Thromb Haemost. 2015;113(6): 1289-1298.

23. Thålin C, Daleskog M, Göransson SP, et al. Validation of an enzyme-linked immunosorbent assay for the quantification of citrullinated histone $\mathrm{H} 3$ as a marker for neutrophil extra- cellular traps in human plasma. Immunol Res. 2017;65(3): 706-712.

24. Nomura K, Miyashita T, Yamamoto Y, et al. Citrullinated histone H3: early biomarker of neutrophil extracellular traps in septic liver damage. J Surg Res. 2019;234:132-138.

25. Douda DN, Jackson R, Grasemann H, Palaniyar N. Innate immune collectin surfactant protein D simultaneously binds both neutrophil extracellular traps and carbohydrate ligands and promotes bacterial trapping. J Immunol. 2011;187(4): 1856-1865.

26. Gaberel T, Montagne A, Lesept F, et al. Urokinase versus alteplase for intraventricular hemorrhage fibrinolysis. Neuropharmacology. 2014;85:158-165.

27. Dang G, Yang Y, Wu G, et al. Early erythrolysis in the hematoma after experimental intracerebral hemorrhage. Transl Stroke Res. 2017;8(2):174-182.

28. Hua Y, Schallert T, Keep RF, et al. Behavioral tests after intracerebral hemorrhage in the rat. Stroke. 2002;33(10): 2478-2484.

29. Zamanian JL, Xu L, Foo LC, et al. Genomic analysis of reactive astrogliosis. J Neurosci. 2012;32(18):6391-6410.

30. Chen Z, Gao C, Hua Y, et al. Role of iron in brain injury after intraventricular hemorrhage. Stroke. 2011;42(2):465-470.

31. Mirzadeh Z, Doetsch F, Sawamoto K, et al. The subventricular zone en-face: wholemount staining and ependymal flow. $J$ Vis Exp. 2010(39).

32. Laridan E, Denorme F, Desender L, et al. Neutrophil extracellular traps in ischemic stroke thrombi. Ann Neurol.2017; 82(2):223-232.

33. Garton T, Keep RF, Wilkinson DA, et al. Intraventricular hemorrhage: the role of blood components in secondary injury and hydrocephalus. Transl Stroke Res. 2016;7(6):447-451.

34. Sahlas DJ, Gould L, Swartz RH, et al. Tissue plasminogen activator overdose in acute ischemic stroke patients linked to poorer functional outcomes. J Stroke Cerebrovasc Dis. 2014; 23(1):155-159.

35. Ong C-T, Wong Y-S, Wu C-S, Su Y-H. Outcome of stroke patients receiving different doses of recombinant tissue plasminogen activator. Drug Des Devel Ther. 2017;11:1559-1566.

36. Malinova V, Schlegel A, Rohde V, Mielke D. Catheter placement for lysis of spontaneous intracerebral hematomas: does a catheter position in the core of the hematoma allow more effective and faster hematoma lysis? Neurosurg Rev. 2017; 40(3):397-402.

37. Kannemeier C, Shibamiya A, Nakazawa F, et al. Extracellular RNA constitutes a natural procoagulant cofactor in blood coagulation. Proc Natl Acad Sci U S A. 2007;104(15): 6388-6393.

38. Morrissey JH, Choi SH, Smith SA. Polyphosphate: an ancient molecule that links platelets, coagulation, and inflammation. Blood. 2012;119(25):5972-5979.

\section{Disclosures}

The authors report no conflict of interest concerning the materials or methods used in this study or the findings specified in this paper.

\section{Author Contributions}

Conception and design: Xie, Tan, Xian, Feng. Acquisition of data: Xie, Tan, Guo, Wang. Analysis and interpretation of data: Guo, Wang, Zeng, Liang. Administrative/technical/material support: Chen, Yu. Study supervision: Chen, Yu.

\section{Correspondence}

Zhi Chen: Southwest Hospital, Third Military Medical University (Army Medical University), Chongqing, China. zhichen@tmmu. edu.cn. 